\title{
OPEN GEO-SPATIAL DATA FOR SUSTAINABLE FOREST MANAGEMENT: LITHUANIAN CASE
}

\author{
*Daiva Tiškutè-Memgaudiené ${ }^{1}$, Gintautas Mozgeris ${ }^{1}$, Algis Gaižutis ${ }^{2}$ \\ ${ }^{1}$ Vytautas Magnus University, Lithuania \\ ${ }^{2}$ Forest Owners Association of Lithuania, Vilnius University, Lithuania \\ *Corresponding author's email: daiva.tiskute-memgaudiene@vdu.lt
}

\begin{abstract}
In Lithuania, forests are managed by Lithuanian State Forest Enterprise, municipalities, ministries, etc. and private forest owners. About $50 \%$ of all forest land is State importance, privately owned forests cover $40 \%$ of forest land, and about $10 \%$ of forest land belongs to forests reserved for restitution. Forest management of private ownership force many challenges, because private forest owners are people, who have purchased or received the property after restitution, and often lacks knowledge about forest resources, its dynamics and sustainable forest management. As remote sensing is a valuable source for forest monitoring, because it provides periodic data on forest resource and condition status, these methods are gaining increased attention worldwide. In this context, more scientific efforts are made at developing remote sensing derived geo-spatial data services for sustainable forest management through a web service platform, which would integrate geo-information into daily decision making processes and operation for private forest owners. This article presents a review of privately owned forests' statistics, questionnaire-based survey about GIS usage and demand for forest owners in Lithuania and links available sources of open geo-spatial data useful for sustainable forest management.
\end{abstract}

Key words: private forests owners, open geo-spatial data.

\section{Introduction}

The importance of providing remote sensing and other geo-spatial data for sustainable forest management is increasing persistently (Pasanen et al., 2005; Kivinen et al., 2008; Store \& Antikainen, 2010; Brožová et al., 2020; Shang et al., 2020). Today, forest data and especially remote sensing-derived data can be generated more quickly than we can process it, and it could be integrated into daily decision making processes. Data, provided by the European Union (EU) under the framework of the Copernicus programme, as well as global Earth Observation data from Landsat satellite under joint NASA/USGS program, provides opportunities to wide extent, content and duration land monitoring programs. Operationalization of airborne laser scanning and hyperspectral imaging is also increasing (Wulder et al., 2008; Eysn et al., 2012; Tang \& Shao, 2015; Yrttimaa et al., 2020; Tusa et al., 2020). Despite the supply, demand for geo-spatial data remains limited. Even more, limited are the solutions to turn the massive data in-flows into useful geospatial information, thus, limited are competences and the needs of potential geo-spatial data users.

In scientific society, efforts of developing and making available sophisticated algorithms for remote sensing data processing, gets more attention (Chen et al., 2020; Mi \& Chen, 2020; Sabat-Tomala et al., 2020; Sothe et al., 2020; Zhang et al., 2020). It is hard to expect that average forest stakeholder gets into sophisticated algorithms for remote sensing data processing. Actually, there is no need to understand them, but such algorithms can be implemented into daily decision making process. Thus, there is a potential demand of platform where numerous users could get geo-spatial data-derived solutions without penetrating into sophisticated technologies of satellite or aircraft-based data collection and processing. Such a platform to be functional, it must be user-oriented. On the other hand, the potential user of the platform needs to understand and know how his/her needs can be met better, cheaper, more efficiently, also be able to think about new needs.

With this study, we aim to review the statistics of privately owned forests in Lithuania and link them with demands for geo-spatial data, bearing in mind the needs of private forestry. We emphasize available open sources of geo-spatial data both at national and the European Union (EU) levels, suitable for solving specific forest management tasks.

\section{Materials and Methods}

To review the statistics and spatial pattern of privately owned forests in Lithuania, methods of spatial analysis, descriptive and spatial statistics analysis, utilizing ArcGIS and MS EXCEL software, were used. Due to changes in the state forestry administration system, the inventory of private forest land parcels has changed. Thus, geodatabase of the State Forest Cadastre, available from the State Forest Service, representing the forest cover status on Jan 1, 2017, was used in this study. To evaluate spatial pattern of private, reserved for restitution and other forests cover, the Jenks optimization method was applied. Jenks optimization method is data clustering method, which determines the best arrangement of data values into different classes, minimizing each class's average deviation from the class mean and maximizing each class's deviation from the means of the other groups. The polygons, corresponding to management areas of former state enterprise, were used in the spatial 
pattern analysis of private forests cover. A spatial autocorrelation analysis was performed using global Moran's I statistics. Further, Anselin Local Morans index (cluster and outlier analysis) was used to determine which analyzed polygons have significant clustering. As a result of spatial pattern analysis, choropleth map was developed.

In addition, the method of questionnaire was used to collect and evaluate the data of private forest owners' knowledge about geo-spatial data and GIS software usage in Lithuanian private forest sector. This questionnaire was not the main part of this study and just aimed to get acquainted with the situation of GIS usage by private forest owners in Lithuania. Questionnaires were mailed to 80 private forest owner companies. The questionnaire-based survey was conducted in 2019 March - April, in which 31 respondents from 21 municipalities of Lithuania took part.

Finally, we reviewed open geo-spatial data, available at national and EU levels, aiming to simulate the applications related to sustainable forest management. Open geo-spatial data sources were linked to suitability for solving specific forest management task with access using expert evaluation.

\section{Results and Discussions}

As reported by official statistics of Lithuania (Source: Official statistics portal), forest land area according to Forest Assessment covered 2189.6 thous. ha of Lithuanian territory in 2017. This means that forests covered $33.5 \%$ (Figure 1).
More than half of forest area, i.e. 50.4\%, were owned privately or reserved for restitution and $49.6 \%$ of forest area was state importance.

The forest cover, within different state forest enterprises boundaries, is clustered (Global Moran's Index 0.617, z-score 5.042, p-value 0.000) (Figure 2).

Privately owned forest cover was discovered to vary from $16.6 \%$ to $79.6 \%$ in 2017 . The modest, i. e. $16.6 \%$, privately owned forest cover was found in the management area of Kazlų Rūda training state forest enterprise (SFE). Slightly larger private forest cover was found in the management area of Dubrava experimental and training SFE (24.5\%), Joniškis SFE (25.9\%) and Marijampolè SFE (29.9\%). Analysis of spatial pattern of private, reserved for restitution and other forests cover, has revealed that in most, i. e. 27 , analyzed polygons privately owned forest cover vary from 32.7-55.9\%. Largest areas of privately owned forests were found in 11 analyzed polygons, where the private forest cover varied from $58.5 \%$ in the management area of Tytuvenai SFE to $71.1 \%$ in the management area of Zarasai SFE and even $79.6 \%$ in the management area of Utena SFE. Such high percent of privately owned forest in the management area of Zarasai SFE and the management area of Utena SFE, could be determined by relief with predominated lake landscape and high demand of forests encompassing recreational use. Despite enlarged private forest cover, because of recreational demand in Utena and Zarasai districts, results of analysis of privately owned forest cover distribution in Lithuania ascertains that private forest estates cover large area of Lithuania territory,

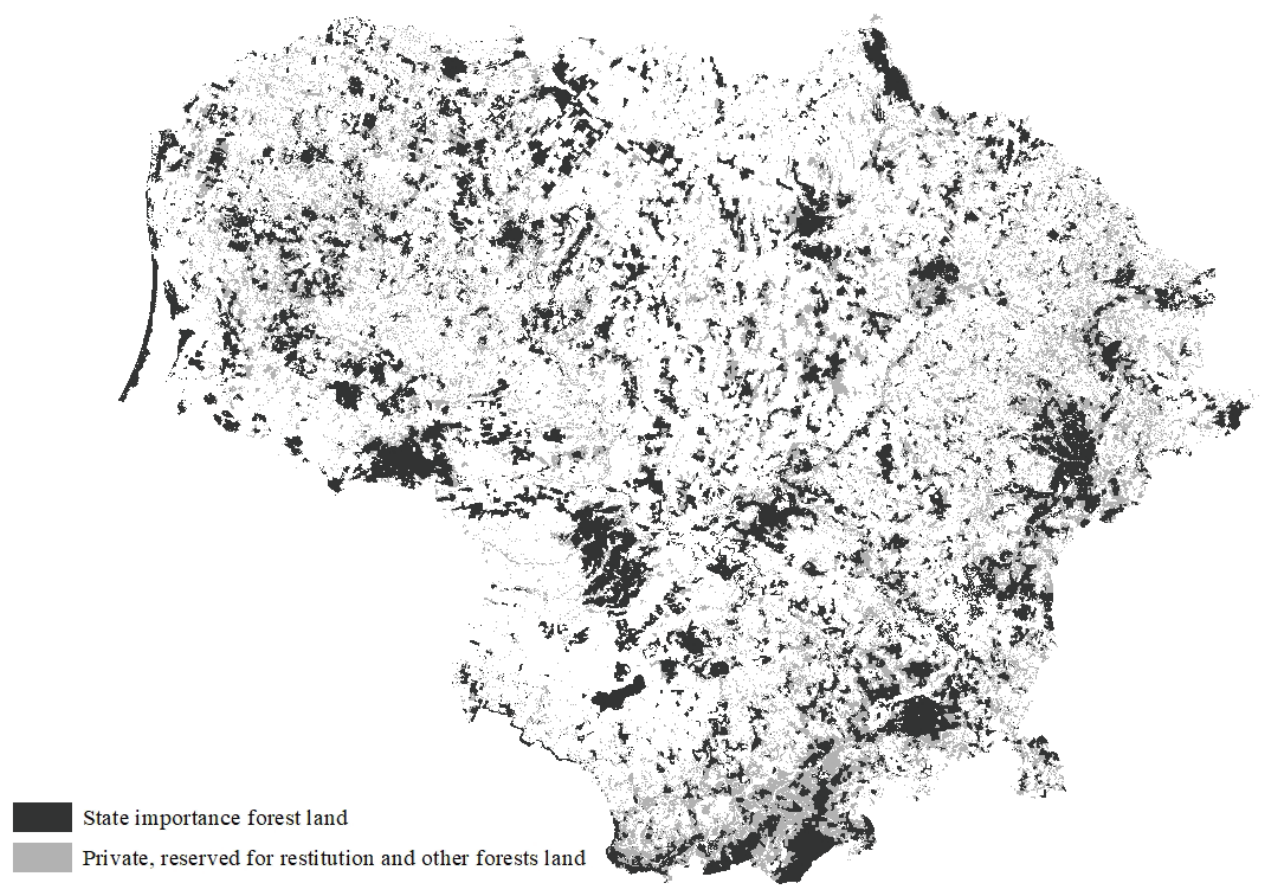

Figure 1. Lithuanian forests by ownership types in Lithuania (Source: State Forest Cadastre, 2017). 


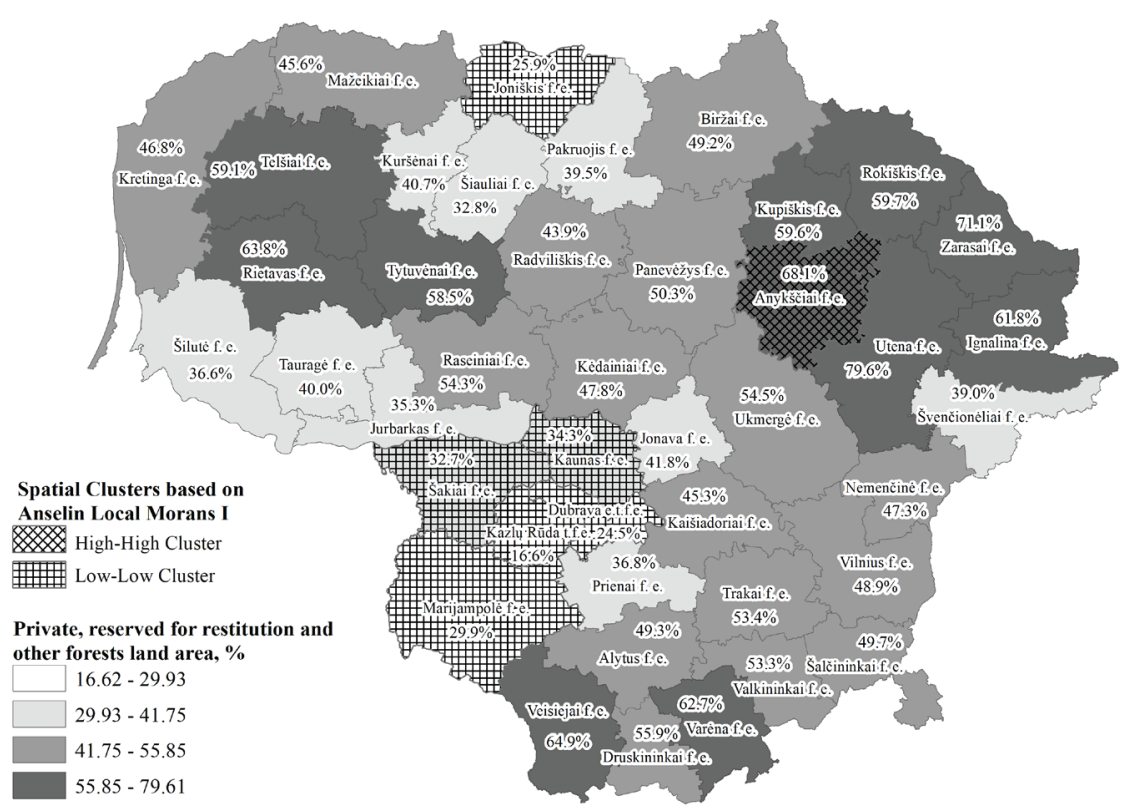

Figure 2. Spatial pattern of private, reserved for restitution and other forests (i.e. not the forests of state importance), within state forest enterprises boundaries, in Lithuania.

involving lots of private owners with more or less, or even no knowledge about forestry and sustainable forest management.

The questionnaire-based survey has indicated that just $32 \%$ of private forest companies used GIS technology in the daily professional activities. $20 \%$ of the respondents, if needed, bought GIS services and $10 \%$ of respondents planned to start using GIS in coming 3 years. Private forest owners used two different software for geo-spatial data analysis and mapping, e. g. commercial software ArcGIS (60\%) and open source software QGIS (40\%). The following reasons why GIS was not used by private forest owners, where listed: lack of demand (44\%), high hardware and software costs $(41 \%)$, lack of professional GIS users in private forest sector $(9 \%)$, lack of geo-spatial data $(6 \%)$. The questionnaire-based survey ascertains that usage of geo-spatial data as well as software for such data processing is fragmented among private forest owners in Lithuania. As it could be foreknown, private forest owners, who manage about half of Lithuania forests, lack innovative, thus sustainable, instruments and data in their daily decision-making activities and long-term strategical process.

Further study has revealed, that there is lots of open geo-spatial data sources covering Lithuania territory and providing data for forest inventory and management, starting with Georeference spatial data set of the territory of the Republic of Lithuania at scale 1:250 000 according to the requirements of the international project EuroRegionalMap and Spatial data set of (geo) reference base cadastre (GRPK), continuing with additional data for forest management as Data of state cadastre for protected areas of Lithuania, Bogs and peatlands of Lithuania, Natural habitats of EU importance, General restrictions of forest management in NATURA 2000 sites, etc. and ending with key data for forest management as Forest cadastre of Lithuania where data about forest management plans in private and state forests, forest's conditions after natural events (fires, windstorms), forest compartments, land utilities, etc. are provided. Moreover, some data is provided in different data formats which are used for both - open source software as QGIS, e. g. GML and commercial software as ArcGIS, e. g. ESRI FileGeodatabase, ESRI Shape, etc. In addition, users of this portal can use not only Inspire download service (WFS), but also Inspire view service (WMS) - both types of services are standardized by Open Geospatial Consortium (OGC) (Table 1).

Main open geo-spatial information providing data about land cover as well as forests at European Union level can be accessed by European Environment Agency (EEA) under the framework of the Copernicus programme. Some iterative open geo-spatial data adjusted for Lithuania territory can be found in geoportal of Lithuania (Table 2).

Remote sensing and forest management has long been associated in Lithuania only in the context of forest inventory related application (Bikuviene \& Tiškutè-Memgaudienė, 2016; Mozgeris, 2008; Mozgeris et al., 2008). Stand-wise forest inventories were completely based on application of orthophotos, 
Table 1

\section{Open geo-spatial data available at national level}

\begin{tabular}{|c|c|c|c|c|}
\hline Name of open geo-spatial data & $\begin{array}{l}\text { Service type in } \\
\text { geoportal.lt }\end{array}$ & Author & Data format & $\begin{array}{c}\text { Forest management } \\
\text { tasks the data could } \\
\text { be used for }\end{array}$ \\
\hline Forest cadastre data & $\begin{array}{l}\text { Dataset, Inspire } \\
\text { view service, } \\
\text { Inspire download } \\
\text { service }\end{array}$ & $\begin{array}{l}\text { State Forest Survey } \\
\text { service under } \\
\text { the Ministry of } \\
\text { Environment }\end{array}$ & $\begin{array}{l}\text { ESRI shape, } \\
\text { WMS, WFS }\end{array}$ & $1,2,4-20^{*}$ \\
\hline Boundaries of state forest areas & $\begin{array}{l}\text { Inspire view } \\
\text { service, Inspire } \\
\text { download service }\end{array}$ & $\begin{array}{l}\text { State Forest Survey } \\
\text { service under } \\
\text { the Ministry of } \\
\text { Environment }\end{array}$ & WMS, WFS & $1,2 *$ \\
\hline $\begin{array}{l}\text { The high resolution layer of } \\
\text { forest }\end{array}$ & Dataset & $\begin{array}{l}\text { Environmental } \\
\text { Protection Agency }\end{array}$ & TIFF & $9,11,14,15^{*}$ \\
\hline $\begin{array}{l}\text { Natural habitats of EU } \\
\text { importance }\end{array}$ & $\begin{array}{l}\text { Dataset, Inspire } \\
\text { view service }\end{array}$ & $\begin{array}{l}\text { The Institute of } \\
\text { Botany of Nature } \\
\text { Research Centre }\end{array}$ & $\begin{array}{l}\text { ESRI } \\
\text { FileGeodatabase, } \\
\text { ESRI Shape, etc., } \\
\text { WMS }\end{array}$ & $2,5,6,11,16-19 *$ \\
\hline $\begin{array}{l}\text { Natural habitats of EU } \\
\text { importance (boundaries) }\end{array}$ & $\begin{array}{l}\text { Inspire view } \\
\text { service }\end{array}$ & $\begin{array}{l}\text { The Institute of } \\
\text { Botany of Nature } \\
\text { Research Centre }\end{array}$ & WMS & $2,5,6,11,16-19^{*}$ \\
\hline $\begin{array}{l}\text { Natural habitats of EU } \\
\text { importance }\end{array}$ & Dataset & SE GIS-Centras & GML & $2,5,6,11,16-19^{*}$ \\
\hline $\begin{array}{l}\text { Bogs and peatlands of Lithuania } \\
\text { ( } 2018 \text { evaluation by LFN) }\end{array}$ & $\begin{array}{l}\text { Inspire view } \\
\text { service }\end{array}$ & $\begin{array}{l}\text { Lithuanian Fund for } \\
\text { Nature }\end{array}$ & WMS & $2,9,11-15^{*}$ \\
\hline $\begin{array}{l}\text { GRPK - Spatial data set of (geo) } \\
\text { reference base cadastre }\end{array}$ & $\begin{array}{l}\text { Inspire view } \\
\text { service, Inspire } \\
\text { download service }\end{array}$ & $\begin{array}{l}\text { Ministry of } \\
\text { Agriculture of } \\
\text { the Republic of } \\
\text { Lithuania }\end{array}$ & WMS, WFS & $1,2,7-20^{*}$ \\
\hline $\begin{array}{l}\text { GRPK - Spatial data set of (geo) } \\
\text { reference base cadastre (static) }\end{array}$ & Dataset & $\begin{array}{l}\text { Ministry of } \\
\text { Agriculture of } \\
\text { the Republic of } \\
\text { Lithuania }\end{array}$ & $\begin{array}{l}\text { ESRI } \\
\text { FileGeodatabase, } \\
\text { ESRI Shape, etc. }\end{array}$ & $1,2,7-20^{*}$ \\
\hline $\begin{array}{l}\text { GRPK - Spatial data set of } \\
\text { (geo) reference base cadastre } \\
\text { (dynamic) }\end{array}$ & Dataset & $\begin{array}{l}\text { Ministry of } \\
\text { Agriculture of } \\
\text { the Republic of } \\
\text { Lithuania }\end{array}$ & $\begin{array}{l}\text { ESRI } \\
\text { FileGeodatabase, } \\
\text { ESRI Shape, etc. }\end{array}$ & $1,2,7-20^{*}$ \\
\hline $\begin{array}{l}\text { GRPK map - Georeferential } \\
\text { Base Map at Scale 1:10 } 000\end{array}$ & Other Services & $\begin{array}{l}\text { Ministry of } \\
\text { Agriculture of } \\
\text { the Republic of } \\
\text { Lithuania }\end{array}$ & PDF & $1,2,7-20^{*}$ \\
\hline $\begin{array}{l}\text { ERM_250LT - georeference } \\
\text { spatial data set of the territory } \\
\text { of the Republic of Lithuania at } \\
\text { scale 1:250 } 000 \text { according to the } \\
\text { requirements of the international } \\
\text { project EuroRegionalMap }\end{array}$ & $\begin{array}{l}\text { Dataset, Inspire } \\
\text { view service }\end{array}$ & $\begin{array}{l}\text { National Land } \\
\text { Service under } \\
\text { the Ministry of } \\
\text { Agriculture }\end{array}$ & $\begin{array}{l}\text { ESRI File } \\
\text { Geodatabase, } \\
\text { WMS }\end{array}$ & $9-14,20^{*}$ \\
\hline $\begin{array}{l}\text { View service of Free State Land } \\
\text { Fund data }\end{array}$ & $\begin{array}{l}\text { Inspire view } \\
\text { service }\end{array}$ & $\begin{array}{l}\text { National Land } \\
\text { Service under } \\
\text { the Ministry of } \\
\text { Agriculture }\end{array}$ & WMS & $14,17,18^{*}$ \\
\hline $\begin{array}{l}\text { Free State land fund information } \\
\text { interactive electronic service }\end{array}$ & $\begin{array}{l}\text { Inspire view } \\
\text { service }\end{array}$ & $\begin{array}{l}\text { National Land } \\
\text { Service under } \\
\text { the Ministry of } \\
\text { Agriculture }\end{array}$ & WMS & $14,17,18^{*}$ \\
\hline
\end{tabular}




\begin{tabular}{|c|c|c|c|c|}
\hline Name of open geo-spatial data & $\begin{array}{l}\text { Service type in } \\
\text { geoportal.lt }\end{array}$ & Author & Data format & $\begin{array}{l}\text { Forest management } \\
\text { tasks the data could } \\
\text { be used for }\end{array}$ \\
\hline $\begin{array}{l}\text { The National atlas of the } \\
\text { Republic of Lithuania in digital } \\
\text { format. Forests }\end{array}$ & $\begin{array}{l}\text { Inspire view } \\
\text { service }\end{array}$ & $\begin{array}{l}\text { National Land } \\
\text { Service under } \\
\text { the Ministry of } \\
\text { Agriculture }\end{array}$ & WMS & $9-11,14,15,20^{*}$ \\
\hline $\begin{array}{l}\text { The National atlas of the } \\
\text { Republic of Lithuania in digital } \\
\text { format. Forest density }\end{array}$ & $\begin{array}{l}\text { Inspire view } \\
\text { service }\end{array}$ & $\begin{array}{l}\text { National Land } \\
\text { Service under } \\
\text { the Ministry of } \\
\text { Agriculture } \\
\end{array}$ & WMS & $9-11,14,15,20^{*}$ \\
\hline $\begin{array}{l}\text { General restrictions of forest } \\
\text { management in NATURA } 2000 \\
\text { sites }\end{array}$ & $\begin{array}{l}\text { Inspire view } \\
\text { service }\end{array}$ & \begin{tabular}{|l|} 
State service for \\
protected areas \\
under the Ministry of \\
Environment \\
\end{tabular} & WMS & $\begin{array}{l}1,2,4-6,9,11-13, \\
15,16-18^{*}\end{array}$ \\
\hline $\begin{array}{l}\text { Data of State Cadastre for } \\
\text { Protected Areas of Lithuania }\end{array}$ & $\begin{array}{l}\text { Dataset, Inspire } \\
\text { view service }\end{array}$ & $\begin{array}{l}\text { State service for } \\
\text { protected areas } \\
\text { under the Ministry of } \\
\text { Environment }\end{array}$ & $\begin{array}{l}\text { ESRI } \\
\text { FileGeodatabase, } \\
\text { ESRI Shape, etc., } \\
\text { WMS }\end{array}$ & $1,2,4-20^{*}$ \\
\hline $\begin{array}{l}\text { Land Parcel Identification } \\
\text { System (KZS) Database }\end{array}$ & Dataset & SE GIS-Centras & GML & $11,14,15,18^{*}$ \\
\hline $\begin{array}{l}\text { Data Base of Limited Land } \\
\text { Use Areas of the Republic of } \\
\text { Lithuania at scale 1:10 } 000 \\
\text { SŽNS_DR10LT }\end{array}$ & $\begin{array}{l}\text { Dataset, Inspire } \\
\text { view service }\end{array}$ & $\begin{array}{l}\text { National Land } \\
\text { Service under } \\
\text { the Ministry of } \\
\text { Agriculture }\end{array}$ & $\begin{array}{l}\text { ESRI } \\
\text { FileGeodatabase, } \\
\text { WMS }\end{array}$ & $1,2,4-20^{*}$ \\
\hline $\begin{array}{l}\text { Data Base of Limited Land } \\
\text { Use Areas of the Republic of } \\
\text { Lithuania at scale 1:10 } 000 \\
\text { SŽNS_DR10LT (dynamically } \\
\text { updated) SŽNS_DR10LT }\end{array}$ & Dataset & $\begin{array}{l}\text { National Land } \\
\text { Service under } \\
\text { the Ministry of } \\
\text { Agriculture }\end{array}$ & ESRI Shape & $1,2,4-20^{*}$ \\
\hline $\begin{array}{l}\text { Data Base of Limited Land } \\
\text { Use Areas of the Republic of } \\
\text { Lithuania at scale 1:10000 } \\
\text { SŽNS DR10LT }\end{array}$ & Dataset & SE GIS-Centras & GML & $1,2,4-20^{*}$ \\
\hline
\end{tabular}

*1 - forest inventory; 2 - forest management planning; 3 - monitoring of silviculture activities; 4 - afforestation/reforestation planning; 5 - final harvesting planning; 6 - thinning planning; 7 - forest logistics; 8 - forest fire protection; 9 - environment protection; 10 - recreation planning; 11 - biodiversity assessment; 12 - wildlife management; 13 - game management; 14 - land use change monitoring; 15 - wetland management; 16 - economic evaluation; 17 - real estate management; 18 EU subsidies management; 19 - pest control; 20 - historical heritage.

while the National forest inventory by sampling methods utilized also some alternative sources of remotely sensed information, including satellite images (Kasperavičius et al., 2000). The procurement of stand-wise forest inventories in the country was mainly associated with the update of the Forest State Cadaster, and the inventories were implemented on a 10-years cycle for all forests in the country, i.e. no matter the ownership. However, the reform in administration of state forests in Lithuania which took place in 2018 (Makrickiene et al., 2019) resulted in stopping unified and state paid stand-wise inventories in the private forests. Nevertheless, to implement forest management activities, all forest owners are required to have valid forest management plans, preceded by field inventories, implemented usually by numerous individual forest inventory engineers.
It is obvious, that the private forest owner possessing several hectares of forest may not pay for remotely sensed data acquisition missions, even though they are very cheap solution based on e.g. the use of unmanned aviation vehicles or ultra-light aviation (Mozgeris \& Augustaitis, 2013; Mozgeris et al., 2018a; Mozgeris et al., 2018b). Thus, availability of open source data opens additional opportunities for private forest inventories and management planning, even though the data may be not perfectly emulating the conventional orthophotos.

Nowadays, management of private forests in Lithuania does not necessary include aiming for profits, usually arriving from timber harvesting (Stanislovaitis et al., 2015). In that context, we see additional uses of open data sources for tasks currently not considered widely in current forest 
Table 2

\section{Open geo-spatial data available at EU level}

\begin{tabular}{|c|c|c|c|c|c|}
\hline $\begin{array}{l}\text { Name of open geo- } \\
\text { spatial data }\end{array}$ & Resource type & Author & Data format & $\begin{array}{c}\text { Spatial } \\
\text { resolution, } \\
\mathrm{m}\end{array}$ & $\begin{array}{c}\text { Forest management } \\
\text { tasks the data could } \\
\text { be used for } *\end{array}$ \\
\hline $\begin{array}{l}\text { CORINE land } \\
\text { cover (CLC) } 1995\end{array}$ & \multirow{4}{*}{$\begin{array}{l}\text { Dataset, Inspire } \\
\text { view service, } \\
\text { Inspire download } \\
\text { service }\end{array}$} & \multirow{5}{*}{$\begin{array}{l}\text { Environmental } \\
\text { Protection } \\
\text { Agency }\end{array}$} & \multirow{4}{*}{$\begin{array}{l}\text { ESRI File Geodatabase, } \\
\text { ESRI Personal } \\
\text { Geodatabase, ESRI } \\
\text { Shape, WMS, WFS }\end{array}$} & \multirow[t]{4}{*}{-} & \multirow[t]{4}{*}{$9-15,19^{*}$} \\
\hline $\begin{array}{l}\text { CORINE land } \\
\text { cover (CLC) } 2000\end{array}$ & & & & & \\
\hline $\begin{array}{l}\text { CORINE land } \\
\text { cover (CLC) } 2006\end{array}$ & & & & & \\
\hline $\begin{array}{l}\text { CORINE land } \\
\text { cover (CLC) } 2012\end{array}$ & & & & & \\
\hline $\begin{array}{l}\text { CORINE land } \\
\text { cover (CLC) } 2018\end{array}$ & $\begin{array}{l}\text { Dataset, Inspire } \\
\text { view service }\end{array}$ & & $\begin{array}{l}\text { ESRI File Geodatabase, } \\
\text { ESRI Personal } \\
\text { Geodatabase, ESRI } \\
\text { Shape, WMS }\end{array}$ & & \\
\hline $\begin{array}{l}\text { CORINE land } \\
\text { cover change } \\
\text { (CHA) 1995-2000 }\end{array}$ & \multirow{2}{*}{$\begin{array}{l}\text { Dataset, Inspire } \\
\text { view service, } \\
\text { Inspire download } \\
\text { service }\end{array}$} & \multirow[t]{3}{*}{$\begin{array}{l}\text { Environmental } \\
\text { Protection } \\
\text { Agency }\end{array}$} & \multirow{2}{*}{$\begin{array}{l}\text { ESRI File Geodatabase, } \\
\text { ESRI Personal } \\
\text { Geodatabase, ESRI } \\
\text { Shape, WMS, WFS }\end{array}$} & \multirow[t]{3}{*}{-} & \multirow[t]{3}{*}{$9-15,19 *$} \\
\hline $\begin{array}{l}\text { CORINE land } \\
\text { cover change } \\
\text { (CHA) 2000-2006 }\end{array}$ & & & & & \\
\hline $\begin{array}{l}\text { CORINE land } \\
\text { cover change } \\
(\mathrm{CHA}) 2006-2012\end{array}$ & $\begin{array}{l}\text { Dataset, Inspire } \\
\text { view service }\end{array}$ & & $\begin{array}{l}\text { ESRI File Geodatabase, } \\
\text { ESRI Personal } \\
\text { Geodatabase, ESRI } \\
\text { Shape, WMS }\end{array}$ & & \\
\hline $\begin{array}{l}\text { CORINE land } \\
\text { cover (CLC) } 1990\end{array}$ & \multirow[t]{9}{*}{ Dataset } & \multirow{9}{*}{$\begin{array}{l}\text { European } \\
\text { Environment } \\
\text { Agency }\end{array}$} & \multirow{9}{*}{$\begin{array}{l}\text { ESRI Geodatabase, } \\
\text { SQLite Database, } \\
\text { GeoTIFF }\end{array}$} & \multirow[t]{9}{*}{100} & \multirow[t]{9}{*}{$9-15,19 *$} \\
\hline $\begin{array}{l}\text { CORINE land } \\
\text { cover (CLC) } 2000\end{array}$ & & & & & \\
\hline $\begin{array}{l}\text { CORINE land } \\
\text { cover (CLC) } 2006\end{array}$ & & & & & \\
\hline $\begin{array}{l}\text { CORINE land } \\
\text { cover (CLC) } 2012\end{array}$ & & & & & \\
\hline $\begin{array}{l}\text { CORINE land } \\
\text { cover (CLC) } 2018\end{array}$ & & & & & \\
\hline $\begin{array}{l}\text { CORINE land } \\
\text { cover change } \\
(\mathrm{CHA}) 1990-2000 \\
\end{array}$ & & & & & \\
\hline $\begin{array}{l}\text { CORINE land } \\
\text { cover change } \\
(\mathrm{CHA}) 2000-2006 \\
\end{array}$ & & & & & \\
\hline $\begin{array}{l}\text { CORINE land } \\
\text { cover change } \\
\text { (CHA) 2006-2012 }\end{array}$ & & & & & \\
\hline $\begin{array}{l}\text { CH CORINE } \\
\text { land cover change } \\
\text { (CHA) A 2012-- } \\
2018\end{array}$ & & & & & \\
\hline $\begin{array}{l}\text { Tree Cover Density } \\
2012\end{array}$ & \multirow[t]{2}{*}{ Dataset } & \multirow{2}{*}{$\begin{array}{l}\text { European } \\
\text { Environment } \\
\text { Agency }\end{array}$} & \multirow[t]{2}{*}{ GeoTIFF } & \multirow[t]{2}{*}{20} & \multirow[t]{2}{*}{$9-11,14,15,20^{*}$} \\
\hline $\begin{array}{l}\text { Tree Cover Density } \\
2015\end{array}$ & & & & & \\
\hline
\end{tabular}




\begin{tabular}{|c|c|c|c|c|c|}
\hline $\begin{array}{c}\text { Name of open geo- } \\
\text { spatial data }\end{array}$ & Resource type & Author & Data format & $\begin{array}{l}\text { Spatial } \\
\text { resolution, } \\
\mathrm{m}\end{array}$ & $\begin{array}{l}\text { Forest management } \\
\text { tasks the data could } \\
\text { be used for } *\end{array}$ \\
\hline $\begin{array}{l}\text { Tree Cover Density } \\
\text { Change 2012-2015 }\end{array}$ & Dataset & $\begin{array}{l}\text { European } \\
\text { Environment } \\
\text { Agency }\end{array}$ & GeoTIFF & 100 & $9-11,14,15,20 *$ \\
\hline $\begin{array}{l}\text { Dominant Leaf } \\
\text { Type } 2012\end{array}$ & \multirow[t]{2}{*}{ Dataset } & \multirow{2}{*}{$\begin{array}{l}\text { European } \\
\text { Environment } \\
\text { Agency }\end{array}$} & \multirow[t]{2}{*}{ GeoTIFF } & \multirow[t]{2}{*}{20} & \multirow[t]{2}{*}{$\begin{array}{l}8,9,11,12,14,15 \\
19^{*}\end{array}$} \\
\hline $\begin{array}{l}\text { Dominant Leaf } \\
\text { Type } 2015\end{array}$ & & & & & \\
\hline Forest Type 2012 & \multirow[t]{2}{*}{ Dataset } & \multirow{2}{*}{$\begin{array}{l}\text { European } \\
\text { Environment } \\
\text { Agency }\end{array}$} & \multirow[t]{2}{*}{ GeoTIFF } & \multirow[t]{2}{*}{20,100} & \multirow{2}{*}{$\begin{array}{l}8,9,11,12,14,15 \\
19^{*}\end{array}$} \\
\hline Forest Type 2015 & & & & & \\
\hline $\begin{array}{l}\text { Forest Additional } \\
\text { Support Layer } \\
2012\end{array}$ & \multirow[t]{2}{*}{ Dataset } & \multirow[t]{2}{*}{$\begin{array}{l}\text { European } \\
\text { Environment } \\
\text { Agency }\end{array}$} & \multirow[t]{2}{*}{ GeoTIFF } & \multirow[t]{2}{*}{20} & \multirow[t]{2}{*}{$\begin{array}{l}3,8,9,11,12,14 \\
15,19^{*}\end{array}$} \\
\hline $\begin{array}{l}\text { Forest Additional } \\
\text { Support Layer } \\
2015\end{array}$ & & & & & \\
\hline
\end{tabular}

*1 - forest inventory; 2 - forest management planning; 3 - monitoring of silviculture activities; 4 -afforestation/reforestation planning; 5 - final harvesting planning; 6 - thinning planning; 7 - forest logistics; 8 - forest fire protection; 9 - environment protection; 10 - recreation planning; 11 - biodiversity assessment; 12 - wildlife management; 13 - game management; 14 - land use change monitoring; 15 - wetland management; 16 - economic evaluation; 17 - real estate management; 18 EU subsidies management; 19 - pest control; 20 - historical heritage.

management practices. Such data may become a corner stone to develop and introduce a cost and time efficient solution for permanent monitoring of private (or all forests no matter the ownership) forests, including detection of natural and human induced changes, permanent updating data required for stand-wise forest inventories, supporting improved forest management decisions and advice. Obviously, historical information on the conditions of forest resources is a prerequisite to start monitor the development. Moreover, using open GIS data sets provide more information needed to quantify other forest ecosystem services and conduct the monitoring of them in Lithuanian private forest estates, e.g. the carbon balance, regulatory forest services, including reduced mortality risks due to wind, insects or diseases or providing habitats for forest dwelling species (Bāders et al., 2018a; Bāders et al., 2018b; Treinys et al., 2016).

Available datasets seem to cover large diversity of needs for sustainable management of private forests in Lithuania. Thus, availability of data may hardly be considered as the factor restricting progress of geospatial technologies in private forestry in Lithuania. This is also confirmed by relatively low share $(6 \%)$ of respondents, thinking that geo-spatial technologies are purely used in private forest sector because of geo-spatial data lack. Therefore, we explain the relatively low level of GIS utilization in private forest management in the country by limited needs for taking of enhanced management decisions. We explain this also by strong impacts of command and control forest management, inherited from the planned economy (Makrickiene et al., 2019).

\section{Conclusions and Proposals}

Although privately owned forests cover about half of forest area in Lithuania, slightly small part of private forest owners used GIS technology in their daily professional activities. The list of reasons why GIS technologies were not used by private forest owners is as follows: lack of demand (44\%), high hardware and software costs $(41 \%)$, lack of professional GIS users in private forest sector (9\%), lack of geo-spatial data (6\%). But our study indicated that available open geospatial data covers a large diversity of needs for sustainable management of private forests in Lithuania. GIS technologies could be evolved to an integrated and flexible system, used in all stages of forest inventory, management and control not only in state, but also in private forestry. There is no limitation to implement innovative solutions based on geo-spatial information into sustainable forest management for private forest owners.

\section{Acknowledgements}

This study has been implemented in the context of the European Union's Horizon 2020 research and innovation programme under the grant agreement No. 776045. https://mysustainableforest.com. 


\section{References}

Bāders, E., Jansons, Ā., Matisons, R., Elferts, D., \& Desaine, I. (2018a). Landscape diversity for reduced risk of insect damage: a case study of Spruce bud Scale in Latvia. Forests. 9, 545. DOI: 10.3390/f9090545.

Bāders, E., Lūkins, M., Zariņš, J., Krišāns, O., Jansons, Ā., \& Jansons, J. (2018b). Recent land cover changes in Latvia. Proceedings of $24^{\text {th }}$ Annual International Scientific Conference 'Research for Rural Development 2018'. 1, 34-39. DOI: 10.22616/RRD.24.2018.005.

Bikuvienè, I., \& Tiškutè-Memgaudienè, D. (2016). GIS in Lithuanian forest inventory - 20 years' experience. Sinteza 2016 - International Scientific Conference on ICT and E-Business Related Research. $208-212$. DOI: 10.15308/Sinteza-2016-208-212.

Brožová, N., Fischer, J.-T., Bühler, Y., Bartelt, P., \& Bebi, P. (2020). Determining forest parameters for avalanche simulation using remote sensing data. Cold Regions Science and Technology. 172. DOI: 10.1016/j. coldregions.2019.102976.

Chen, D., Loboda, T.V., \& Hall, J.V. (2020). A systematic evaluation of influence of image selection process on remote sensing-based burn severity indices in North American boreal forest and tundra ecosystems. ISPRS Journal of Photogrammetry and Remote Sensing. 159, 63-77. DOI: 10.1016/j.isprsjprs.2019.11.011.

Eysn, L., Hollaus, M., Schadauer, K., \& Pfeifer, N. (2012). Forest Delineation Based on Airborne LIDAR Data. Remote Sensing. 4(3), 762-783. DOI: 10.3390/rs4030762.

Kasperavičius, A., Kuliešis, A., \& Mozgeris, G. (2000). Satellite imagery based forest resource information and its application for designing the national forest inventory in Lithuania. Conference on remote sensing and forest monitoring proceedings. 50-58.

Kivinen, S., Koivisto, E., Keski-Saaria, S., Poikolainen, L., Tanhuanpää, T., Kuzmin, K., Viinikka, A., Heikkinen, R.K., Pykälä, J., Virkkala, R., Vihervaara, P., \& Kumpula, T. (2020). A keystone species, European aspen (Populus tremula L.), in boreal forests: Ecological role, knowledge needs and mapping using remote sensing. Forest Ecology and Management. 462. DOI: 10.1016/j.foreco.2020.118008.

Makrickiene, E., Brukas, V., Brodrechtova, Y., Mozgeris, G., Sedmák, R., \& Šálka, J. (2019). From commandand-control to good forest governance: A critical interpretive analysis of Lithuania and Slovakia. Forest Policy and Economics. 109. DOI: 10.1016/j.forpol.2019.102024.

Mi, L., \& Chen, Z. (2020). Superpixel-enhanced deep neural forest for remote sensing image semantic segmentation. ISPRS Journal of Photogrammetry and Remote Sensing. 159, 140-152. DOI: 10.1016/j. isprsjprs.2019.11.006.

Mozgeris, G. (2008). Estimation and use of continuous surfaces of forest parameters: Options for Lithuanian forest inventory. Baltic Forestry. 14(2), 176-184.

Mozgeris, G., Galaunè, A., \& Palicinas, M. (2008). Systemy informacji geograficznej w urządzaniu lasu na Litwie - dekada praktycznego stosowania (Geographical information systems in forest management planning in Lithuania - a decade of practical application). Sylwan. 152(1), 58-63. (in Polish).

Mozgeris, G., \& Augustaitis, A. (2013). Estimating crown defoliation of Scots pine (Pinus sylvestris L.) trees using small format digital aerial images. iForest-Biogeosciences and Forestry. 6 (1), 15-22. DOI: 10.3832/ ifor0705-006.

Mozgeris, G., Jonikavičius, D., Jovarauskas, D., Zinkevičius, R., Petkevičius, S., \& Steponavičius, D. (2018a). Imaging from manned ultra-light and unmanned aerial vehicles for estimating properties of spring wheat. Precision Agriculture. 19(5), 876-894. DOI: 10.1007/s11119-018-9562-9.

Mozgeris, G., Juodkienė, V., Jonikavičius, D., Straigytė, L., Gadal, S., \& Ouerghemmi, W. (2018b). UltraLight Aircraft-Based Hyperspectral and Colour-Infrared Imaging to Identify Deciduous Tree Species in an Urban Environment. Remote sensing. 10(10), 1-22. DOI: 10.3390/rs10101668.

Official statistics portal Retrieved January 12, 2020, from http://osp.stat.gov.lt/en/statistiniu-rodikliu-analize?p ortletFormName= $=$ isualization $\&$ hash $=3 \mathrm{f} 0702 \mathrm{fa}-845 \mathrm{f}-42 \mathrm{~cd}-9 \mathrm{a} 58-8786 \mathrm{bbfc507c}$.

Pasanen, K., Kurttila, M., Pykäiäinen, J., Kangas, J., \& Leskinen, P. (2005). Mesta - non evaluation of alternative forest plans over the Internet. International Journal of Information Technology \& Decision Making. 04(04), 601-620. DOI: 10.1142/S0219622005001726.

Sabat-Tomala, A., Raczko, E., \& Zagajewski, B. (2020). Comparison of Support Vector Machine and Random Forest Algorithms for Invasive and Expansive Species Classification Using Airborne Hyperspectral Data. Remote Sensing. 12(3), 516. DOI: 10.3390/rs12030516.

Shang, C., Coops, N.C., Wulder, M.A., White, J.C., \& Hermosilla, T. (2020). Update and spatial extension of strategic forest inventories using time series remote sensing and modeling. International Journal of Applied Earth Observation and Geoinformation. 84. DOI: 10.1016/j.jag.2019.101956. 
Sothe, C., De Almeida, C.M., Schimalski, M.B., Liesenberg, V., La Rosa, L.E.C., Castro, J.D.B., \& Feitosa, R.Q. (2020). A comparison of machine and deep-learning algorithms applied to multisource data for a subtropical forest area classification. International Journal of Remote Sensing. 41(5), 1943-1969. DOI: 10.1080/01431161.2019.1681600.

Stanislovaitis, A., Brukas, V., Kavaliauskas, M., \& Mozgeris, G. (2015). Forest owner is more than her goal: a qualitative typology of Lithuanian owners. Scandinavian Journal of Forest Research. 30(5), 478-491. DOI: $10.1080 / 02827581.2014 .998706$.

Store, R., \& Antikainen, H. (2010). Using GIS-based multicriteria evaluation and path optimization for effective forest field inventory. Computers, Environment and Urban Systems. 34(2), 153-161. DOI: 10.1016/j. compenvurbsys.2009.12.003.

Tang, L., \& Shao, G. (2015). Drone remote sensing for forestry research and practice. Journal of forestry research. 26, 791-797.

Tusa, E., Laybros, A., Monnet, J.-M., Mura, M.D., Barré, J.-B., Vincent, G., Dalponte, M., Féret, J.-B., \& Chanussot, J. (2020). Fusion of hyperspectral imaging and LiDAR for forest monitoring. Data Handling in Science and Technology. 32, 281-303. DOI: 10.1016/B978-0-444-63977-6.00013-4.

Treinys, R., Mozgeris, G., \& Skuja, S. (2016). Can intensified forestry be responsible for changes in habitat usage by the forest-dwelling Black Stork? European Journal of Forest Research. 135, 1175-1186. DOI: 10.1007/s10342-016-1003-6.

Wulder, M.A., Bater, C.W., Coops, N.C., Hilker, T., \& White, J.C. (2008). The role of LiDAR in sustainable forest management. The Forestry Chronicle. 84(6), 807-826. DOI: 10.5558/tfc84807-6.

Yrttimaa, T., Saarinen, N., Luoma, V., Tanhuanpää, T., Kankare, V., Liang, X., Hyyppä, J., Holopainen, M., \& Vastaranta, M. (2020). Terrestrial Laser Scanning and Ground Truth Data for Characterizing Downed Dead Wood. OSF. DOI: https://osf.io/rjegd/.

Zhang, J., Hu, X., Dai, H., \& Qu, S.R. (2020). DEM Extraction from ALS Point Clouds in Forest Areas via Graph Convolution Network. Remote Sensing. 12(1), 178. DOI: 10.3390/rs12010178. 